\title{
Tailored Ag nanoparticles/nanoporous superhydrophobic surfaces hybrid devices for the detection of single molecule
}

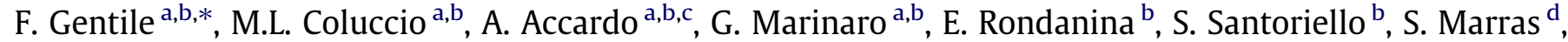

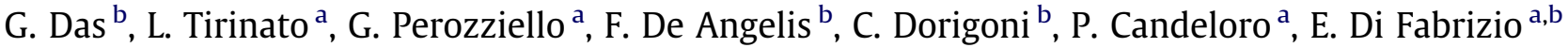 \\ a BioNEM (Bio Nano Engineering and Technology for Medicine), University Magna Graecia of Catanzaro, Catanzaro 88100, Italy \\ ${ }^{\mathrm{b}}$ Nanostructures Department, Italian Institute of Technology, Via Morego, 30, 16163 Genova, Italy \\ 'Soft Matter Structures Group ID13, MICROFOCUS Beamline, European Synchrotron Radiation Facility, Grenoble Cedex, France \\ ${ }^{\mathrm{d}}$ Nanochemistry Department, Italian Institute of Technology, Via Morego, 30, 16163 Genova, Italy
}

\section{A R T I C L E I N F O}

\section{Article history:}

Available online 11 April 2012

\section{Keywords:}

Superhydrophobic surfaces

SERS

Nanoporous silicon

Cancer

\begin{abstract}
A B S T R A C T
Super-hydrophobic surfaces were designed and micro-fabricated. These comprise micro-pillars combined to form a regular hexagonal motif; the pillars were conveniently modified to incorporate nanoporous silicon films. Silver nanoparticles were dispersed into the pores to guarantee superior SERS (surface enhanced Raman scattering) capabilities. On account of its multiscale nature, the substrate exhibits an increased hydrophobicity and a reduced friction coefficient. This device is multiplexed in the sense that features different functions or abilities, including (i) the concentration of diluted solutions, (ii) the separation of 'small' molecules basing on an exclusion principle and (iii) the SERS measurement of these molecules with extreme accuracy. In consideration of the properties above, the device may be employed for investigating systematically the blood serum, and extracting from this the low molecular weight content, that discloses precious information about the ongoing status of tumors or other diseases. Here, analytes were separated and detected in the femto molar $\left(10^{-15} \mathrm{M}\right)$ range.
\end{abstract}

(c) 2012 Elsevier B.V. All rights reserved.

\section{Introduction}

The use of Superhydrophobic surfaces (SHSs) is being recently recognized as fundamental in multifunctional devices that are designed towards the single molecule detection [1-5]. SHSs are typically artificial, micro- or nano-fabricated surfaces, with a texture given by a regular, periodic lattice of cylindrical pillars. The top of pillars may be conveniently modified via either chemical or physical processes (that are, for instance, the deposition of a thin film of polymers as in [1,2], or the formation of a nanoporous silicon - NPSi - matrix as in [4]), and thus the geometry and surface chemistry would combine to yield substrates that are clearly water repellent. A drop of water, positioned upon such a surface, would maintain a quasi spherical shape, with a contact angle at the interface that can be theoretically predicted with excellent accuracy [6-9]. In the celebrated model of Cassie and Baxter [8] the wetting behavior of the surface is lumped in the sole parameter $\phi$, that is the ratio of the solid to the total projected area. When $\phi$ tends to zero, at the interface with the substrate, the liquid mostly 'senses' air and the droplet would resemble a perfect sphere.

* Corresponding author at: Nanostructures Department, Italian Institute of Technology, Via Morego, 30, 16163 Genova, Italy.

E-mail address: gentile@unicz.it (F. Gentile).
The most practical property of our SHSs is their reduced friction coefficient and, on account of this, they can be conveniently used to manipulate diluted solutions of biological or medical interest. SHSs offer realistic possibilities for the detection of extremely low concentrated solutions of analytes. To do this, a small drop of de-ionized water, containing the moieties at study, should be positioned upon the substrate and let it evaporate. In sight of a simple balance of forces, the line of contact at the solid/liquid/air interface would recede with time, and thus the footprint of the drop would also gradually reduce. When the drop gets sufficiently small, the Cassie configuration turns into energetically unfavorable, a transition to a stable state occurs (that is, the Wenzel state [9]), whereby the drop is firmly attached to the substrate, and the scale-down of the area of contact is prevented. Therefore, few molecules would be accumulated into a very small region, assuring an increased density and, accordingly, the attainment of the limits of detection. Noticeably, the use of the sole SHSs is not sufficient for the recognition of single molecule; this technology should be instead incorporated to others in a way that novel multifunctional devices have high selectivity, resolution, and very low detection limits. In $[1,2]$ the simultaneous use of SHSs and nano-geometry based photonic devices was discussed, whereby the former serve as vehicles to concentrate diluted solutions into a small area, and the latter would measure the solute with unprecedented accuracy. In $[4,5]$, 
SHSs and NPSi matrices were combined to yield devices with the capability of concentrating and harvesting small molecules, where the cut-off size can be adequately controlled, with application towards the sensing of few molecules, employing Fourier Transform Infrared (FTIR) spectroscopy.

Here, a novel platform is introduced that combines hierarchically the functions above. Three different devices are integrated into one, and namely, (i) a superhydrophobic, periodic pattern of micro-pillars; (ii) a NPSi film grown upon the pillars and (iii) a randomly distributed cluster of silver nanoparticles (NPs), that partially fills the nanopores (that is, the pores are covered, and not clogged, by the particles). The benefits of this device are threefold, and are secured by its multistage nature. On account of point (i), the device exhibits an increased contact angle and superior nonadhesive properties as described above, diluted solutions would accordingly be manipulated and few molecules concentrated into a limited area; as regarding point (ii), the NPSi film would select the molecules whose size is strictly smaller than a desirable dimension, while the remaining moieties may be disregarded; the silver NPs dispersed in the NPSi matrix, as in (iii), would assure an increased electromagnetic field, thereby Surface Enhanced Raman Scattering (SERS) effects arise and promise spectroscopy measurements with unprecedented accuracy. The process, as a whole, is represented in the cartoon of Fig. 1. The major advance of this device is its capability of combining different structures and functions, including the sensing the low molecular weight content of the blood proteome. Blood is acknowledged to be a formidable, potential source of diagnostic markers for diseases [10-14]. Nevertheless, a number of challenges wait to be faced before blood serum may be used as a routinely screening procedure, including the severe dilution of the peptides of interest, and the interference of abundant proteins. This device, as it stands, offers a promise to face these challenges, basing upon mechanisms such as SERS spectroscopy and an exclusion strategy step.

In this work, 'small' Rhodamine (molecular weight $\sim 500 \mathrm{Da}$ ) molecules were separated, recognized and analyzed, starting from a diluted binary solution containing 'large' bovine serum albumin (BSA) proteins (molecular weight $\sim 60 \mathrm{kDa}$ ). Moieties were detected with a concentration as low as $10^{-15} \mathrm{M}$.

\section{Materials and methods}

Super-hydrophobic surfaces were designed and micro-fabricated. These comprise micro-pillars combined to form a regular hexagonal motif (Fig. 2A); the pillars were conveniently modified as to incorporate NPSi films (Fig. 2B). Silver nanoparticles were dispersed into the pores to guarantee superior SERS capabilities (Fig. 2C). On account of the simultaneous effects of the geometry and the morphology, the device exhibits an increased hydrophobicity, with a contact angle of about $165^{\circ}$ (Fig. 2D).

Drops $(\mathrm{R} \sim 1 \mathrm{~mm})$ of D.I. water containing a 1:1 binary mixture of Rhodamine and Albumin (moles/moles, with initial concentration of $10^{-15} \mathrm{M}$ ) were gently positioned upon the surfaces and left evaporating over time. At the end of the process, few molecules were accumulated in a small region of the plane. The evaporation was performed at room temperature in a clean room to reduce the presence of external contaminants, and lasted approximately $30 \mathrm{~min}$. Prior the full impalement of the drop, the sample was removed by using a pipettor. The residual solute was observed using Raman spectroscopy techniques.

Micro-probed Raman spectra were obtained using Renishaw in via Raman microscope at room temperature through $20 \times / 50 \times$ objectives of a Leica microscope. The Raman spectra were excited by the $514.0 \mathrm{~nm}$ line of an $\mathrm{Ar}^{+}$laser in backscattering geometry. The laser power was hold constant at $0.18 \mathrm{~mW}$ with an integration time of $20 \mathrm{~s}$. The interferograms were recorded with a resolution of $2 \mathrm{~cm}^{-1}$ and were averaged over five samples. Each spectrum was base line corrected with a second degree polynomial function after acquisition. Mapping Raman measurements were performed with step size of 15 and $12.9 \mu \mathrm{m}$ in $\mathrm{x}$ and $\mathrm{y}$-axis direction, respectively.

Rhodamine (R6G) and bovin serum Albumin (BSA) were purchased from Sigma. Deionized water was used for all experiments.
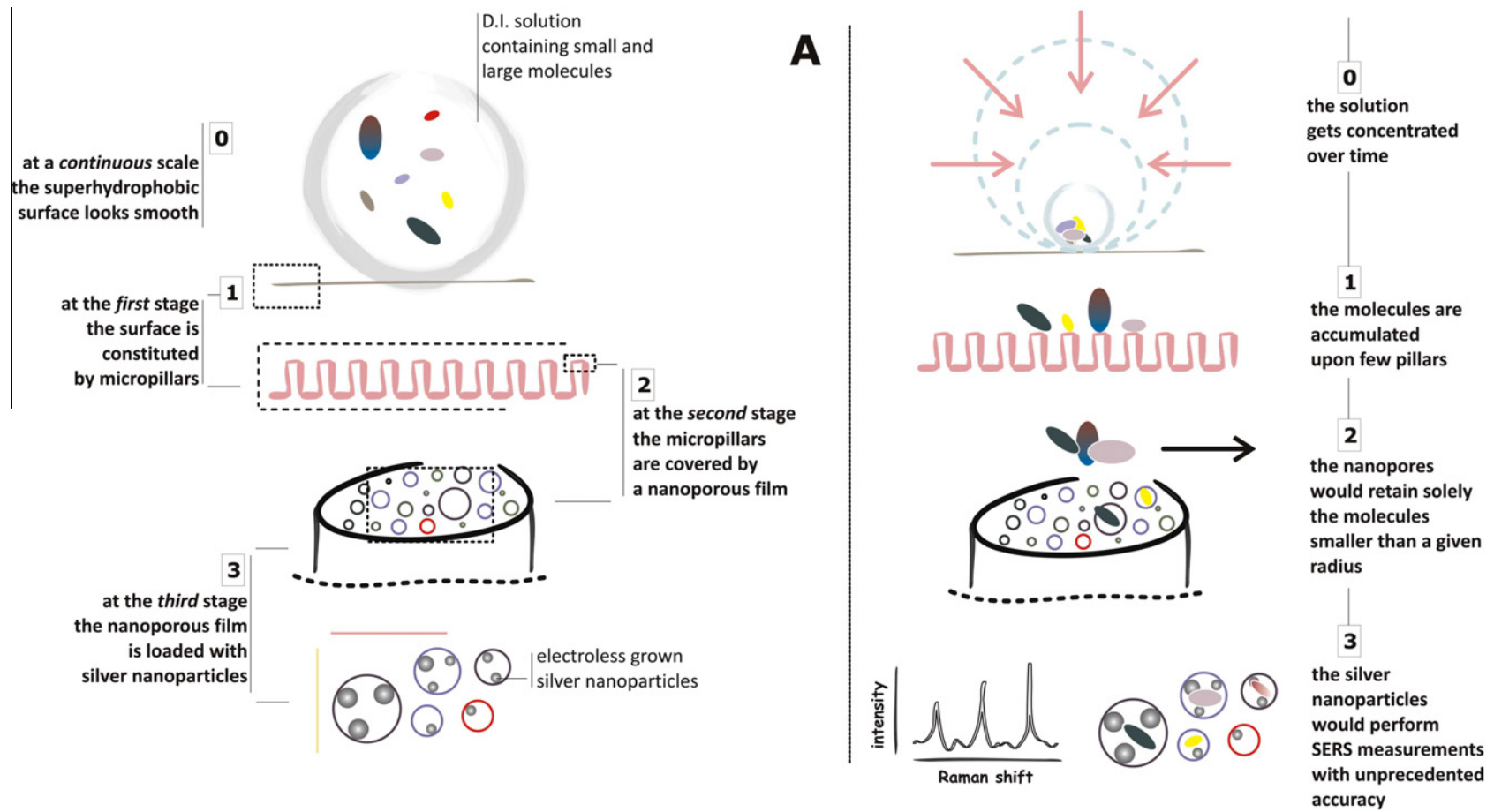

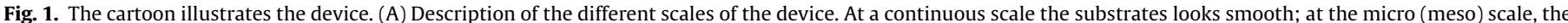

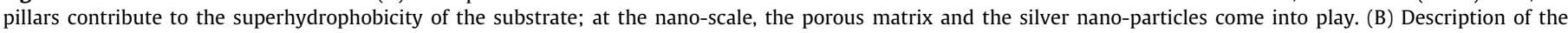
functions the devices is capable of, towards the sensing of few molecules. 

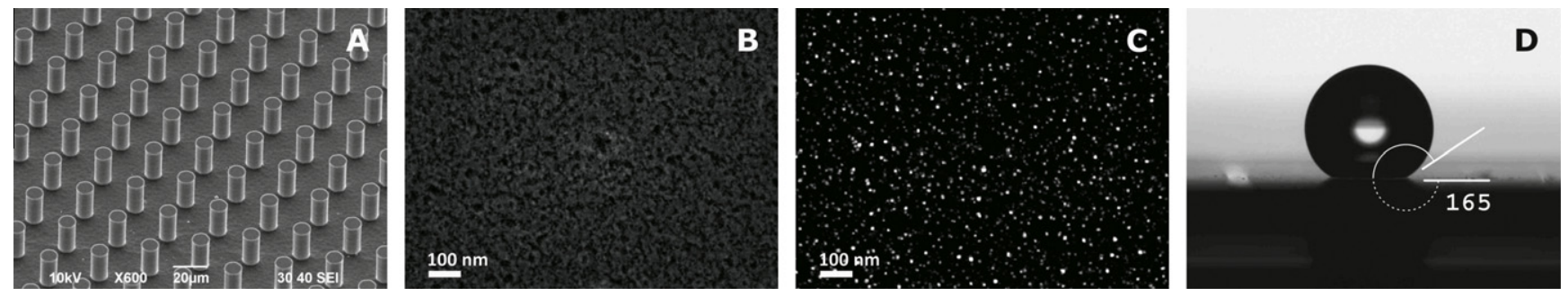

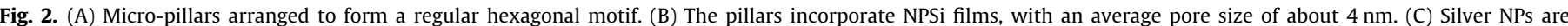

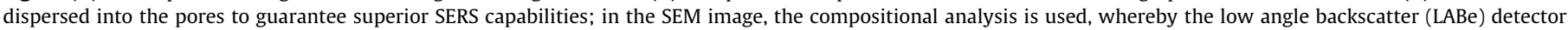

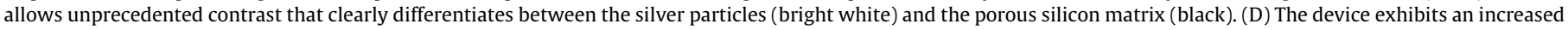
hydrophobicity, with a contact angle approaching $165^{\circ}$.

All chemicals, unless mentioned elsewhere, were of analytical grade and were used as received.

\section{Fabrication}

The device was fabricated on the basis of different steps. Firstly, a surface was obtained comprising a regular hexagonal lattice of $\mathrm{Si}$ cylindrical micro-pillars (Fig. 1A). Secondly, the top of the pillars was conveniently decorated with a NPSi film, this would assure the capability of selecting those molecules whose size is strictly smaller than the pore size that, in the present configuration, is about $4 \mathrm{~nm}$ (Fig. 1B and C). Thirdly, a randomly distributed aggregate of silver NPs was infiltrated through the pores, thus providing the device with superior SERS performance.

Standard optical lithography techniques were employed to realize regular patterns of disks within a layer of negative resist (AZ5214) that was spin-coated onto a clean silicon wafer $(100)$. The disks served as a mask in a Deep Reactive Ion Etching (DRIE) (MESC Multiplex ICP, STS, Imperial Park, Newport, UK) process, whereby the final structures were obtained having a height $h$ of about $20 \mu \mathrm{m}$, a diameter $d$ of $10 \mu \mathrm{m}$ and a pitch $\delta$ of $30 \mu \mathrm{m}$ (that is, $\delta$ is three times $d$ ). These values are consistent with a criterion of optimal design as described, for instance, in [1,2]. From those, it stems out that fraction of solid $\varphi=\pi / 4(d / \delta)^{2}$ settles down to $\pi /$ $36(\sim 0.1)$, that is sufficiently small to assure the superhydrophobicity of the substrate, and still the density of the pillars is adequate to avoid the early collapse of the droplet over large areas.

Upon removal of the residual resist with Piranha solution $\left(\mathrm{H}_{2} \mathrm{O}_{2}: \mathrm{H}_{2} \mathrm{SO}_{4}=1: 3 \mathrm{v} / \mathrm{v}\right)$, porous silicon was obtained by anodization of the boron-doped silicon wafer (resistivity $5-10 \Omega / \mathrm{cm}$ ), using an electrolyte mixture of hydrofluoric acid, water, and methanol $(1: 1: 2, \mathrm{v} / \mathrm{v} / \mathrm{v})$. Forasmuch, a constant current density of $4 \mathrm{~mA} / \mathrm{cm}^{2}$, for $5 \mathrm{~min}$, at room temperature, was applied $[14,15]$. The samples were finally rinsed in D.I. water, ethanol, and pentane. The masks necessary for optical lithography were fabricated using direct laser writing (Heidelberg, DWL66).

Silver nanograin aggregates were implanted to the porous matrix to obtain devices with advanced sensing capabilities. To do so, an electroless growth process was employed, whereby ionic silver was reduced and deposited as metallic silver upon silicon, via a redox reaction. A satisfactory comprehension of the method may be found in [16]. The process was accomplished using a $\mathrm{AgNO}_{3}$ and $\mathrm{HF}$ solution (with a concentration of $0.1 \mathrm{mM}$ and $0.15 \mathrm{M}$, respectively), at a constant temperature of $T=50^{\circ}$, for $60 \mathrm{~s}$. For the present configuration, silver nanograins were obtained with an average dimension strictly smaller than the pore size.

These devices are superhydrophobic on account of their hybrid nature. The micro (that are, the pillars) and the nano (that is, the porous matrix) scales combine hierarchically to yield non-wetting properties, as explained in $[5,17,18]$.

\section{SEM characterization}

Several SEM images of the samples were captured to assure reproducibility and repeatability. A JEOL JSM-7500F scanning electron microscope was used for the measurements. During the acquisitions beam energies of 5 and $15 \mathrm{keV}$, and corresponding electron currents of $0.98 \mathrm{pA}$ and $0.14 \mathrm{nA}$, were used. The silver nanograins were verified using the compositional analysis, whereby the low angle backscatter (LABe) detector allows unprecedented contrast that clearly differentiates between the silver particles and the porous silicon matrix.

\section{Results}

Here, we demonstrated the capability of the device to extract and recognize the small molecular weight information content in a diluted mixture. On that account, a solution was prepared containing a 1:1 binary mixture of Rhodamine and BSA (moles/moles, with initial concentration $10^{-15} \mathrm{M}$ ). A $10 \mu$ droplet was extracted from this solution and gently positioned upon the device. The geometry (that is, the pillars' sizes and their arrangement) and the morphology (that is, the NPSi film decorating the pillars) of the device was combined to yield extremely large contact angles and thus very low friction coefficients (non wetting behavior of SHSs). The evaporation of the drop resulted in a monotonous decrease of the contact area, whereby the molecules dispersed in the drop were conveyed (concentrated) into a small and bounded region of the substrate. The evaporation process was then followed over time. Prior the collapse of the drop (that is, before the irreversible transition to the Wenzel state), the sample was removed by using a pipettor, while the excess of BSA was eluted by immersion in D.I. water.

In Fig. 3A the solute is shown at the final stage of the evaporation process. The deposit is extremely concentrated and partitioned upon few pillars, while in the pillar-pillar interstices there are not solute refuses. On account of the NPSi matrix that decorates the substrate, 'small' molecules are likely to be secured onto the active sites of the device. Here, 'small' would indicate molecules with a characteristic length scale (that is, the hydrodynamic radius $r_{h}$ ) that is less than the pore size. For the present configuration, the average pore size is about $4 \mathrm{~nm}$, and thus R6G molecules $\left(r_{h} \sim 0.5 \mathrm{~nm}\right)$ would be retained, while BSA proteins $\left(r_{h} \sim 6 \mathrm{~nm}\right)$ would not. To assess this, the solute deposit as in Fig. 3A was imaged using SERS techniques. In Fig. $3 \mathrm{~B}$ and $\mathrm{C}$, the intensity profile of R6G molecules is reported in the form of a contour and a 3d plot, respectively, and was derived using, as a reference, a band centered at $1390 \mathrm{~cm}^{-1}$ (Fig. 3D). The retention of R6G molecules is demonstrated by the fact that the Raman signal in Fig. 3B and $C$ follows faithfully the substance distribution as in Fig. 3A. Contrariwise, the SERS intensity map of BSA (Fig. 3E and F shows the reference 

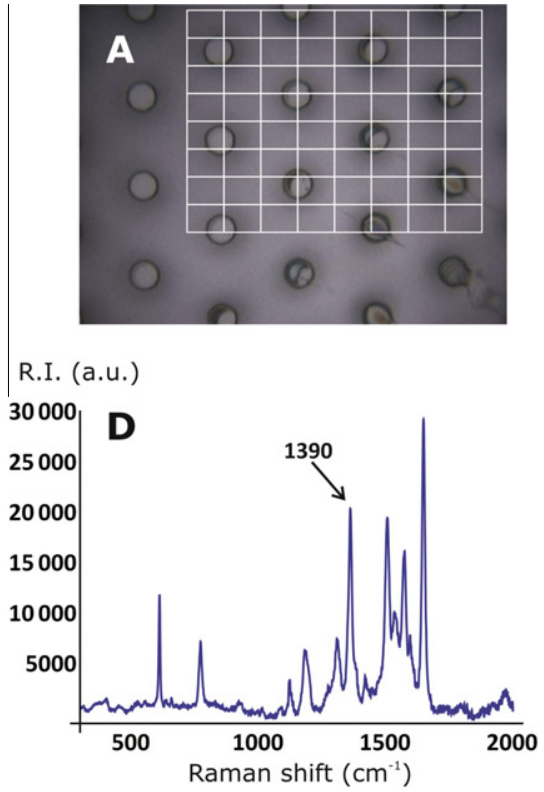
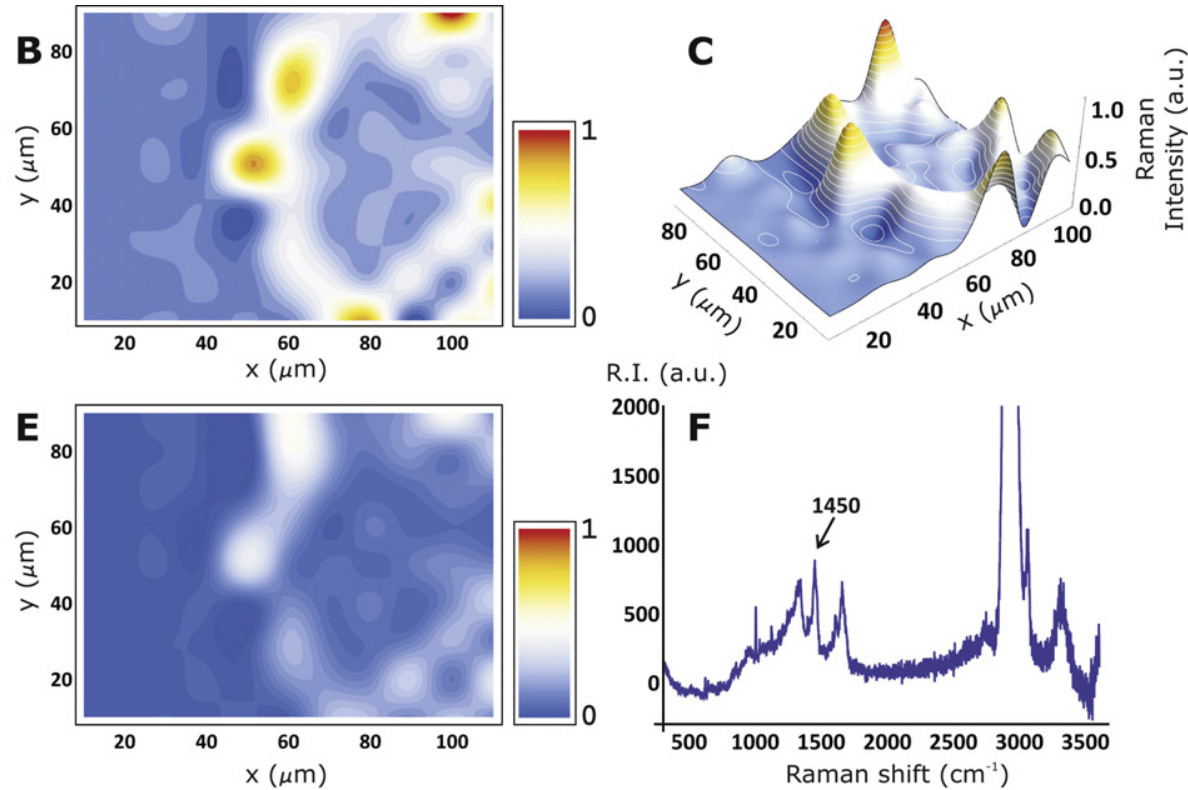

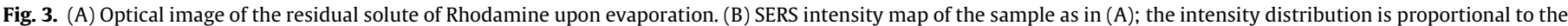

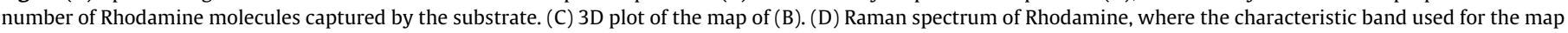

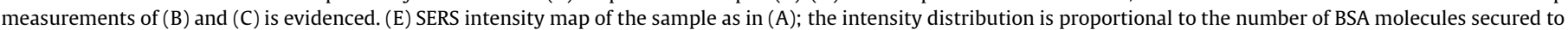

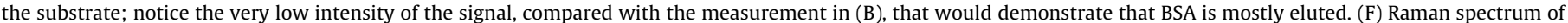
BSA, where the characteristic band used for the map measurements of (E) is evidenced.

frequency for BSA, that is $1450 \mathrm{~cm}^{-1}$ ), is practically trivial everywhere in the region of interest, and thus BSA is largely released. The results reported in Fig. 3, as a whole, would prove that the bio sensor here introduced features high size selectivity and unprecedented sensitivity.

Notice that this technology may be proficiently used in conjunction with others [19-23] to obtain multiplexed platforms with the capability of manipulating, controlling and sensing biological samples. These platforms can size also several centimeters per side, and considering that the area covered by a slowly evaporating droplet is of about $3.14 \mathrm{~mm}^{2}$, a $5 \times 5 \mathrm{~cm}^{2}$ device can be used for some $10^{3}$ times. These systems are thus designed for medium to long-term durability: beyond the promise of detecting cancers, they are also cheap, and may be profitably used in health care.

\section{Conclusions}

Advanced substrates were designed and micro-fabricated. These are multifunctional in that incorporate different functions that only arise due to the heterogeneous scales of the system, and namely the 'micro', on account of which the substrate is superhydrophobic, and the 'nano', that is the typical length scale of the pores and of the silver particles infiltrated through them. In sight of its hierarchical nature, the device may serve as a bio sensor with the ability of (i) concentrating diluted solutions, (ii) selecting the bio molecules of interest and conveying them to the active areas of the device, and thus (iii) analyzing these with extreme accuracy. Node (iii) is, in particular, possible in sight of the silver NPs that yield SERS effects.

Here, small drops of D.I. water containing both low and high molecular weight molecules were conveniently manipulated. The LMW region of the solution was extracted and analyzed. Initial concentrations, as low as $10^{-15} \mathrm{M}$, were detected. These results indicate that this device may be exploited for investigating systematically blood serum, and extracting from that its otherwise inaccessible information content.

\section{Acknowledgements}

This work was funded under European Project SMD FP7-NMP 2800-SMALL-2 (Proposal No. CP-FP 229375-2), Project NANOANTENNA FP7-HEALTH-2009 (Grant Agreement No. 241818), Italian Project FIRB 'Rete Nazionale di Ricerca sulle Nanoscienze ItalNanoNet' (cod. RBPR05JH2P-010) and by the EU Commission, the European Social Fund and the Calabria Region (POR Calabria FSE 2007-2013).

\section{References}

[1] F. Gentile et al., Microelectronic Engineering 87 (2010) 798-801.

[2] F. De Angelis et al., Nature Photonics, DOI: 10.1038/NPHOTON.2011.222, published online: 18 september 2011.

[3] A. Accardo et al., Langmuir 26 (18) (2010) 15057-15064.

[4] F. Gentile et al., Microelectronic Engineering 88 (2011) 1749-1752.

[5] F. Gentile et al., Microelectronic Engineering 88 (2011) 2537-2540.

[6] R. Blossey, Nature Materials 2 (2003) 301-306.

[7] A. Lafuma, D. Quéré, Nature Materials 2 (2003) 457-460.

[8] A.B.D. Cassie, S. Baxter, Transactions of the Faraday Society 40 (1944) 546-551.

[9] R.N. Wenzel, Industrial and Engineering Chemistry 28 (1936) 988-994.

[10] E.M. Posasadas et al., Annals of oncology 16 (2005) 16-22.

[11] R. Etzioni et al., Nature Reviews Cancer 3 (4) (2003) 243-252.

[12] L.A. Liotta, M. Ferrari, E. Petricoin, Nature 425 (6961) (2003) 905

[13] M. Gaspari et al., Journal of Proteome Research 5 (2006) 1261-1266.

[14] G. Das et al., Biosensors and Bioelectronics 24 (2009) 1693-1699.

[15] L. Vaccari et al., Microelectronic Engineering 83 (2006) 1598-1601.

[16] M. Coluccio et al., Microelectronic Engineering 86 (2009) 1085-1088.

[17] M. Nosonovsky, B. Bhushan, Ultramicroscopy 107 (2007) 969-979.

[18] N.A. Patankar, Langmuir 20 (19) (2004) 8209-8213.

[19] V. Garbin et al., Applied Physics Letters 90 (11) (2007) 114103-114105.

[20] M. Tormen et al., Microelectronic Engineering 73 (2004) 535-541.

[21] A.A. Yu et al., Journal of the American Chemical Society 127 (2005) 1677416775.

[22] F. Perennes et al., Journal of Micromechanics and Microengineering 16 (3) (2006) 473-479.

[23] Candeloro P. et al., Analyst DOI: 10.1039/c1an15313g. 